\title{
VALIDACIÓN DE LA VERSIÓN EN ESPAÑOL DE LA ESCALA DE RED SOCIAL LUBBEN-6
}

\author{
VALIDATION OF THE SPANISH VERSION OF THE LUBBEN SOCIAL NETWORK SCALE-6
}

VALIDAÇÃO DA VERSÃO EM ESPANHOL DA ESCALA DE REDE SOCIAL LUBBEN-6

\author{
Mariano Granero', Gastón Perman², Fernando Vazquez Peña ${ }^{3}$, \\ Cynthia Andrea Barbaro ${ }^{4}$, María Estefanía Zozaya ${ }^{5}$, Verónica Samanta Martínez Infantino ${ }^{6}$, \\ Daniela Abdul7, Gimena Soledad Giardini ${ }^{8}$, Roxana Elizabeth Ramos ${ }^{9}$, Sergio Adrián Terrasa ${ }^{10}$
} \begin{abstract}
cantidad y la calidad de los vínculos sociales que tiene una persona.
\section{Conceptos claves:}

¿Qué se sabe sobre el tema?

El aislamiento social es un problema prevalente a nivel global y tiene una fuerte influencia sobre la morbimortalidad de las personas. Si bien existen algunos instrumentos que evalúan el apoyo social, su aplicación suele ser dificultosa en el ámbito clínico. La Escala de Red Social de Lubben-6 (LSNS-6) es una de las más utilizadas por la comunidad internacional y su aplicación es sencilla y breve. Sin embargo, no existen a la fecha trabajos que hayan validado esta escala en el idioma español.
\end{abstract}

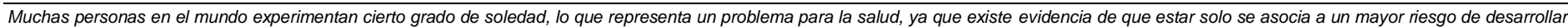
ciertas enfermedades. Por esto, para los profesionales de la salud es muy importante poder medir cuán sola (o no) se encuentra una persona.

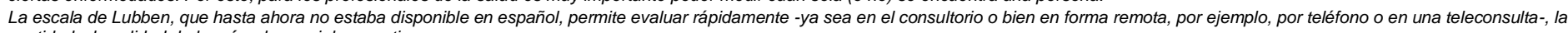

¿Qué aporta este trabajo?

Este trabajo es el primero en traducir, adaptar transculturalmente y validar al español la LSNS-6.

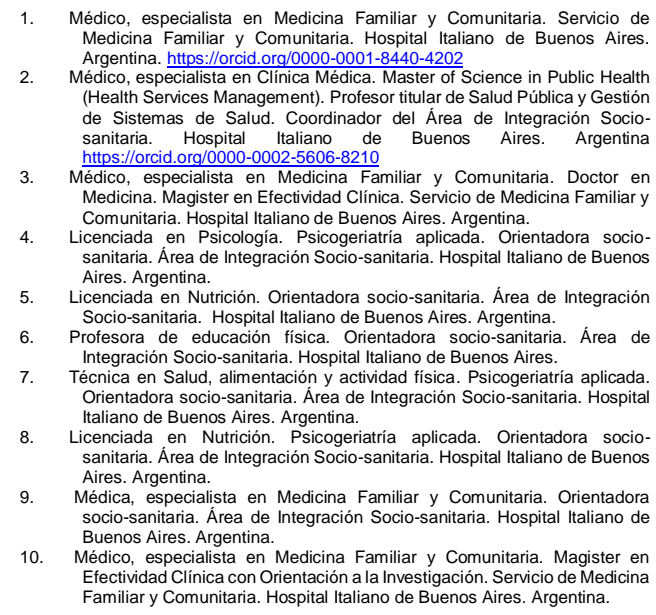

Recibido: 2020-05-26 Aceptado: 2020-06-19

DOI: http://dx.doi.org/10.31053/1853.0605.v77.n4.28775 (c) (i) (8)

CUniversidad Nacional de Córdoba

\section{Resumen:}

Introducción: El apoyo social es un determinante importante de la salud. La Escala de Red Social de Lubben-6 (LSNS-6) es una de las más utilizadas por la comunidad internacional y su aplicación es sencilla y breve. No existen a la fecha trabajos que hayan validado esta escala en el idioma español.

Objetivo: Validar al español la escala LSNS-6.

Métodos: Fue generada una versión en Español del cuestionario a través de la traducción, retrotraducción y adaptación transcultural del LSNS-6.

La validez de constructo fue evaluada mediante un Análisis Factorial Confirmatorio, considerando inicialmente un factor y luego dos. Los resultados fueron comparados mediante indicadores de buen ajuste (IBA). La validez de criterio externo fue evaluada mediante la correlación del puntaje del cuestionario con el asignado por una orientadora sociosanitaria profesional entrenada durante una entrevista presencial en profundidad realizada en el domicilio del paciente.

Resultados: Entrevistamos 150 adultos mayores (54\% hombres; edad media 76,3 años).

Los IBA presentaron valores adecuados para el modelo de dos factores; Chi Cuadrado / Grado de libertad 1,81; "p" ajustada al Chi Cuadrado: 0,069; error de aproximación cuadrático medio (RMSEA) 0,085; Indice de ajuste normado (NFI) 0,97; índice de ajuste no normado (NNFI) 0,99; residuo cuadrático medio estandarizado (RMR st) 0,03.

La correlación Pearson del puntaje de la versión en español del LSNS-6 con el asignado por la orientadora sociosanitaria fue de $0.665(p<0.001)$.

Conclusiones: La versión en español de la escala LSNS-6 es válida y confiable.

Palabras clave: Aislamiento Social; Apoyo social; Determinantes Sociales de la Salud; Estudio de validación

\section{Abstract:}

Introduction: Social support is an important determinant of health. The Lubben-6 Social Network Scale (LSNS-6) is one of the most used by the international community and its application is simple and brief. To date there are no works that have validated this scale in the Spanish language.

Aim of the Study: Validate the spanish version of the LSNS-6.

Methods: The Spanish version of the questionnaire was generated through the translation, back-translation and transcultural adaptation of the LSNS-6, made with native translators and adapted by a bilingual committee. The construct validity was evaluated using a Confirmatory Factor Analysis. We first considered a single factor (family or friends), and then two factors (family and friends). We compared results using Goodness-of-fit index (GFI). The validity of external criteria was evaluated by correlating the score of the questionnaire with that assigned by a professional counselor trained during an in-depth in-person interview conducted at the patient's home.

Results: We interviewed 150 older adults, $54 \%$ men; mean age 76.3 years.

The GFI presented adequate values for the two-factor model $(\mathrm{P}$ Chi2 $=0.069 ; \mathrm{NFI}=0.97 ; \mathrm{NNFI}=0.99$; RMR st. = 0.03; the RMSEA showed a marginal value, 0.085); While the Pearson correlation was 0.665 ( $p$ $<0.001)$.

Conclusion: The Spanish version of the LSNS-6 scale is valid and reliable.

Key words: Social Isolation; Social Support; Social Determinants of Health; Validation study

\section{Resumo}

Introdução: O apoio social é um importante determinante da saúde. A Escala de Rede Social Lubben-6 (LSNS-6) é uma das mais utilizadas pela comunidade internacional e sua aplicação é simples e breve. Até o momento, não existem trabalhos que validaram essa escala no idioma espanhol.

Objetivo do estudo: Valide a versão em espanhol do LSNS-6.

Métodos: A versão em espanhol do questionário foi gerada através da tradução, retrotradução e adaptação transcultural do LSNS-6, feita com tradutores nativos e adaptada por um comitê bilíngue. A validade do construto foi avaliada por meio de uma análise fatorial confirmatória. Primeiro, consideramos um único fator (família ou amigos) e depois dois fatores (família e amigos). Comparamos os resultados usando o índice de qualidade de ajuste (GFI). A validade dos critérios externos foi avaliada correlacionando o escore do questionário com o atribuído por um conselheiro profissional de saúde, durante uma entrevista em pessoa, realizada em casa.

Resultados: Entrevistamos 150 idosos, 54\% homens; idade média de 76,3 anos.

$\mathrm{O}$ GFI apresentou valores adequados para o modelo de dois fatores (P Chi2 = 0,069; NFI = 0,97; NNFI = 0,99; RMR st. = 0,03; o RMSEA apresentou valor marginal, 0,085); Enquanto a correlação de Pearson foi de $0,665(p<0,001)$

Conclusão: A versão em espanhol da escala LSNS-6 é válida e confiável.

Palavras-chave: Isolamento Social; Apoio Social; Determinantes Sociais da Saúde; Estudo da Validação 


\section{Introducción}

Existe evidencia creciente de que el Apoyo Social tiene una fuerte asociación con la morbimortalidad. Un metaanálisis realizado en 2010 1 que incluyó 148 estudios longitudinales (308.849 participantes, con una media de edad de 64 años) comunicó que las personas con mala red social tienen una probabilidad de morir 50\% mayor que aquellos individuos con una buena red social (OR 1,50 IC 95\% 1,42 a 1,59). De esta manera, el aislamiento social tiene una influencia sobre la morbimortalidad similar a otras variables bien conocidas como el

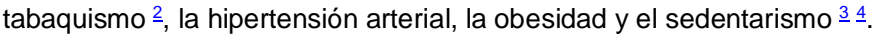
Las personas con aislamiento social tienen más enfermedad cardiovascular y depresión $\stackrel{5-7}{ }$, mayor discapacidad, respuestas más pobres frente a la enfermedad y muertes más tempranas $\underline{8} \stackrel{9}{.} \mathrm{La}$ evaluación del apoyo social de las personas es, por lo tanto, de gran relevancia 4 .

Si bien existen algunos instrumentos validados al español que evalúan el Apoyo Social 10 , su aplicación en el ámbito clínico ambulatorio suele ser dificultosa dado que son muy extensos y consumen mucho tiempo de la consulta médica.

La Escala de Red Social de Lubben (en inglés Lubben Social Network Scale: LSNS-6) fue desarrollada para evaluar el Apoyo Social percibido de un adulto mayor 11 en función del tamaño de su red social, de la confianza que estos vínculos generan y de la frecuencia de los contactos que tiene esta persona con quienes la rodean. La LSNS-6 incluye seis preguntas divididas en dos dominios: tres sobre la familia y tres sobre amigos. Es una de los más utilizadas por la comunidad internacional y su aplicación es sencilla y breve, siendo esto de vital importancia para que sea viable su aplicación en el ámbito clínico. Además, puede ser administrada por cualquier persona con mínimo entrenamiento.

Si bien esta escala ya fue traducida y validada en distintas lenguas $\underline{12-}$ 16, no contábamos con una versión en español, por lo que comunicamos los resultados de la traducción, adaptación transcultural y validación de la LSNS-6 al español.

\section{Objetivo}

Traducir al español, adaptar transculturalmente y validar la escala LSNS-6.

\section{Métodos}

\section{Diseño General}

El equipo investigador que realizó el trabajo en terreno estuvo conformado por un equipo de Evaluación Socio-Sanitaria y un médico de cabecera (investigador principal del estudio).

\section{1) Equipo de Evaluación Socio-Sanitaria}

Éste funciona desde hace cinco años en el Hospital Italiano de Buenos Aires y tiene como objetivo optimizar los cuidados de los pacientes del ámbito ambulatorio que, por distintas comorbilidades, se encuentran confinados en sus domicilios. Cuando un paciente ingresa en este programa, de forma rutinaria una de las orientadoras socio-sanitarias realiza en el domicilio del paciente una entrevista en profundidad de 90 minutos de duración en la que evalúa la red de apoyo del paciente (estructural y percibido), la coordinación de los cuidados que el paciente requiere, el manejo de su medicación crónica, el manejo de dinero y el riesgo de caídas.

A los efectos de este trabajo de investigación, luego de que realizara su tarea habitual (descrita en el párrafo anterior), a cada orientadora socio-sanitaria se le pidió que respondiera una pregunta adicional respecto de los pacientes evaluados y que aceptaron participar de esta investigación:

"Teniendo en cuenta los aspectos objetivos y subjetivos de la interacción social, ¿que puntaje del 1 al 10 le asignaría a la red social del paciente evaluado, considerando que 1 representa la ausencia de apoyo social y 10 representa una red de apoyo social ideal?

Se había consensuado previamente con las orientadoras sociosanitarias que por "red de apoyo social ideal" se entendería a una red de apoyo amplia ( 2 o más miembros), presente (de fácil acceso en caso de ser necesitada), continente y suficiente (que pudiera proveer ayuda en caso de que el paciente lo necesitara); y que sus intervenciones se llevaran a cabo dentro de un marco que preservara la autonomía del paciente. A fin de minimizar la variabilidad interobservador en esta escala, antes de comenzar con la ejecución de este trabajo nuestro equipo realizó dos encuentros con las orientadoras socio-sanitarias en los que se generaron consensos sobre potenciales problemas de interpretación y aplicación de esta escala y sobre cómo sortearlos. Posteriormente, se realizó un tercer encuentro en el que se realizaron dos simulacros con casos clínicos.

Finalmente, y luego de que le asignara dicha valoración del 1 al 10 a la red social del paciente, se le pidió a la orientadora que aplicara el cuestionario LSNS-6 al paciente evaluado.

\section{2) Médico de cabecera (investigador principal)}

Este médico de cabecera realiza habitualmente su tarea asistencial en un consultorio del ámbito ambulatorio, donde reclutó pacientes que cumplieran con los criterios de inclusión. A quienes aceptaron participar, les aplicó el LSNS-6 luego de que finalizara su consulta programada.

\section{Población}

La escala fue administrada a un total de 150 adultos mayores de 65 años de ambos sexos, atendidos en el ámbito ambulatorio del Hospital Italiano de Buenos Aires, que tenían el español como lengua nativa o al menos 30 años de residencia en Argentina, y que aceptaron participar del estudio. Fue criterio de exclusión la presencia de deterioro cognitivo moderado o severo.

Se realizó un muestreo de conveniencia. Para garantizar que la muestra tuviera una buena representación de personas con escasa red social fueron incluidas 100 personas confinadas a su domicilio que eran visitados por una orientadora socio-sanitaria. Las otras 50 personas incluidas en la muestra fueron entrevistadas en el ámbito ambulatorio por el investigador principal.

\section{Generación y validación de la escala}

A partir de un diseño de corte transversal, nuestro trabajo se realizó en etapas (ver figura 1):
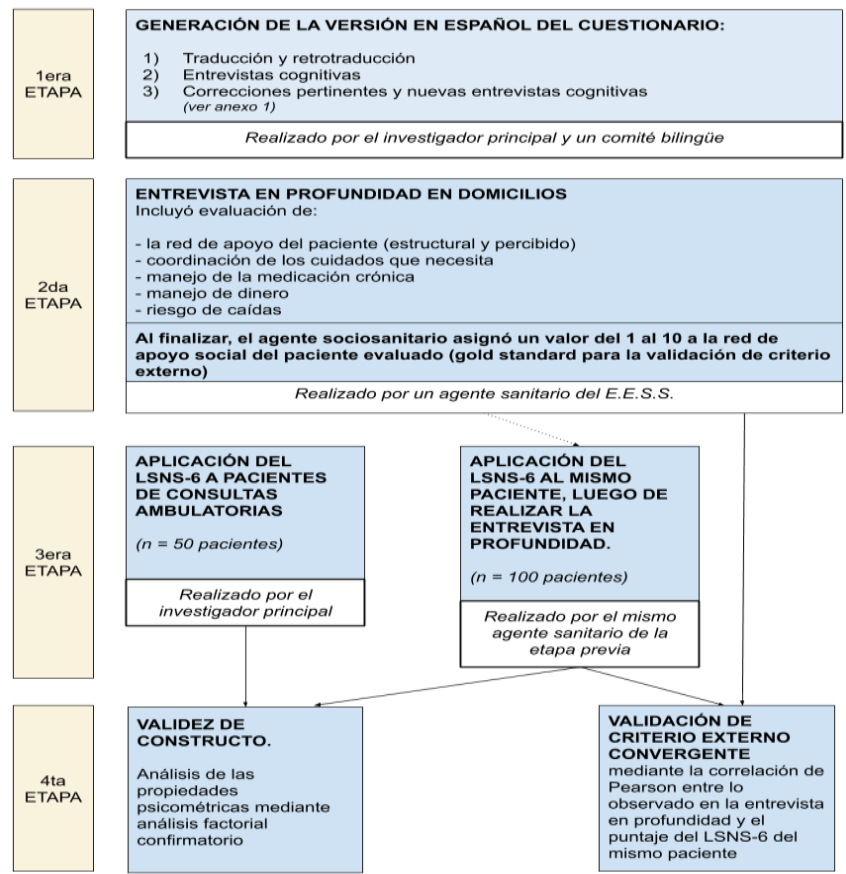

Figura 1. Representación gráfica de las etapas, tareas desarrolladas, y personas a cargo en este trabajo de investigación. 


\section{Traducción y adaptación transcultural de la escala}

Se realizó la traducción al castellano de la escala LSNS-6 a través de dos traductores que tenían al castellano como lengua nativa y formación en medicina, y que generaron individualmente dos versiones en español. Ambas versiones fueron conciliadas por estos dos traductores. La versión resultante fue luego retro-traducida al inglés por dos traductores que no conocían la escala original. Estos últimos tenían al inglés como lengua nativa, uno de ellos con formación en medicina y el otro en ciencias sociales. La escala final resultante de este proceso fue evaluado por todo el comité bilingüe, que corrigió mínimos errores de puntuación 17 .

Esta versión fue puesta a prueba a través de ocho entrevistas cognitivas realizadas por el investigador principal a pacientes que cumplían con los criterios de selección utilizados posteriormente en este trabajo (personas mayores de 65 años, sin deterioro cognitivo y que hablaran el español como lengua nativa, atendidos en el ámbito ambulatorio del Hospital Italiano de Buenos Aires). Fueron realizadas bajo el formato de "pensar en voz alta" $\underline{18}$, en las que los entrevistados transmitían las dificultades que encontraban para entender el contenido de la escala.

Durante estas entrevistas documentamos que algunas personas tenían dificultades para saber a quién llamar "amigo", por lo que el grupo investigador decidió agregar en el encabezado de las preguntas incluidas en los dominios "amigos" y "familiares" una definición aclaratoria respecto de a quiénes se podía considerar amigos y a quienes familiar (ver aclaraciones en el anexo $\mathrm{n}^{\circ} \mathbf{1}$ ).

Esta nueva versión fue chequeada a través de otras ocho entrevistas cognitivas, en las que verificamos que la comprensión de la escala era adecuada.

La versión final de la escala se describe en el anexo 1.

\section{Validez de constructo}

Debido a que la escala original había sido desarrollada en el extranjero, para analizar la validez de constructo de la versión en español, utilizamos Análisis Factorial Confirmatorio (AFC) con el objetivo de certificar la estructura bidimensional (dos factores) de la escala. Dado que la misma cuenta con menos de 15 ítems (seis ítems), basándonos en la propuesta de Brown y col. $\underline{19}$, estimamos que sería necesario contar con una muestra de 150 observaciones.

Aplicamos el estimador robusto de máxima verosimilitud (en inglés Robust Maximum Likelihood) debido a que la escala de Lubben-6 cuenta con ítems ordinales de seis categorías y la distribución de sus resultados no presentó normalidad multivariada. Fue utilizado el programa Lisrel. Consideramos inicialmente un solo factor que incluye ambos dominios (familia $y$ amigos), y luego los dos factores por separado (familia $O$ amigos). Comparamos los resultados mediante indicadores de buen ajuste (IBA) $\underline{20}$.

La fiabilidad fue evaluada a través de la fiabilidad compuesta (FC) de cada dominio y de la varianza media extractada (VME) $\stackrel{21}{1}$, verificando que la fiabilidad compuesta sea de por lo menos 0,7 y la varianza media extractada de $0,5 \underline{22}$

Para evaluar la validez convergente (VC) verificamos que las cargas factoriales estandarizadas en los modelos finales fueran todas mayores a 0,6 ó 0,7 , y con significancia estadística (valores de $t>1.96) \underline{23}$.

Para determinar la validez discriminante (VD), tuvimos en cuenta que la varianza que cada dominio compartiera con sus indicadores propios fuera mayor a la que pudiera compartir con otros dominios incluidos en el modelo. De este modo, documentamos que los valores de la Varianza Media Extractada (VME) de cada dominio fueran mayores que el cuadrado de las correlaciones entre ese dominio y cada uno de los otros.

\section{Validación de criterio externo convergente}

Como patrón para evaluar si nuestra escala realmente medía lo que se supone debía medir, tomamos como prueba de referencia (gold standard) a la valoración de la red social de los 100 pacientes que realizó la orientadora socio-sanitaria.

Una vez obtenida la calificación de la red social por parte de la orientadora socio-sanitaria se realizó el análisis de la validez de criterio externo a través de la correlación Pearson entre la valoración de la red social del paciente por parte de la orientadora socio-sanitaria y el puntaje de la escala LSNS-6 contestado por el participante en cuestión. En este caso según la bibliografía especializada el valor debería estar entre 0,3 y $0,7 \underline{24}$

\section{Aspecto éticos}

El presente trabajo fue evaluado y aprobado por el Comité de Ética de Protocolos de Investigación (CEPI) del Hospital Italiano de Buenos Aires (número de expediente: \#3413)

\section{Resultados}

\section{$\underline{\text { Traducción y adaptación transcultural }}$}

A lo largo de esta etapa del proceso, las discrepancias fueron resueltas en reuniones del equipo investigador. No fue necesario eliminar o agregar ítems, por lo que la escala resultante en español contiene la misma cantidad de preguntas que el original en inglés. La mayoría de los ítems de la escala original tuvieron una traducción literal al español, necesitándose solamente modificaciones en la redacción de algunas preguntas.

La principal dificultad que surgió durante la realización de las entrevistas cognitivas estuvo vinculada a lo que los entrevistados entendían por "amigo/a", dado que consideraban que algunas personas formaban parte de su red de apoyo, pero sin tener la cercanía necesaria para ser consideradas amigos (por ejemplo, vecinos de su cuadra, portero del edificio, sacerdote del barrio, etc.).

Dada esta dificultad, el equipo investigador consensuó aclarar en la escala que podía considerarse amigo a toda persona que formara parte de su red de apoyo (por ejemplo, un vecino) pero que no tuviera un parentesco (sanguíneo o político) con el entrevistado.

\section{Validación de constructo y fiabilidad}

Fue entrevistada una muestra de 150 personas, 81 (54\%) varones y 69 (46\%) mujeres. Su edad promedio fue 76,3 años.

Como muestran la tabla 1 y la figura 2, verificamos la estructura unifactorial y de dos factores de la escala.

Tabla 1. Indicadores del análisis factorial confirmatorio de la versión adaptada al español de la escala de apoyo social de Lubben-6.

\begin{tabular}{|l|l|l|l|l|l|l|}
\hline Modelos & $\begin{array}{l}\text { Chi2 } \\
\text { grados de } \\
\text { libertad(a) } \\
\left(\begin{array}{l}\text { valores de } \\
\text { referencia) }\end{array}\right.\end{array}$ & $\begin{array}{l}\boldsymbol{p} \text { (valor } \\
\text { ajustado)(b) } \\
(>0,05)\end{array}$ & $\begin{array}{l}\text { RMSEA(c) } \\
(<0.08=\text { bueno, } \\
\text { entre } 0.08 \text { y } 0.1= \\
\text { mediocre } \\
y>0,1=\text { malo })\end{array}$ & $\begin{array}{l}\text { NFI(d) } \\
(>0,95)\end{array}$ & $\begin{array}{l}\text { NNFI(e) } \\
(>0,95)\end{array}$ & $\begin{array}{l}\text { RMR } \\
\text { st.(f) } \\
(<0,08)\end{array}$ \\
\hline $\begin{array}{l}\text { Un } \\
\text { factor }\end{array}$ & $115,25 / 9=12,8$ & 0,0000 & 0,396 & 0,78 & 0,64 & 0,29 \\
\hline $\begin{array}{l}\text { Dos } \\
\text { factores }\end{array}$ & $14,53 / 8=1,81$ & 0,069 & 0,085 & 0,97 & 0,99 & 0,03 \\
\hline
\end{tabular}

- Índices evaluados con su valor esperado:
a) Chi Cuadrado / Grado de libertad;
b) "p" ajustada del test Chi Cuadrado (Satorra-Bentler);
c) RMSEA: error de aproximación cuadrático medio;
d) NFI: Indice de ajuste normado;
e) NNFI: índice de ajuste no normado;
f) RMR st: residuo cuadrático medio estandarizado.
- Todos los valores recomendados que figuran en esta tabla fueron extraídos de Brown 2015
19. 


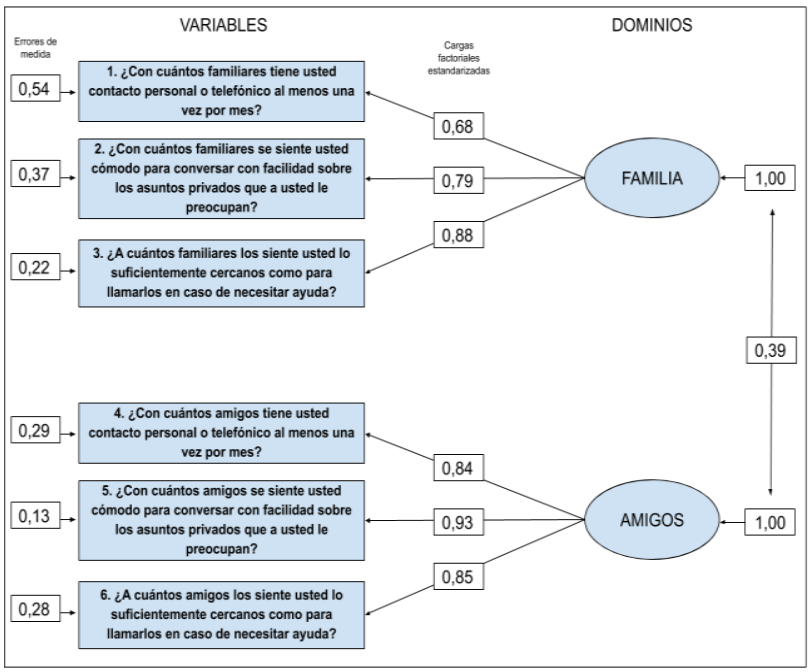

Figura 2. Representación gráfica de los resultados del análisis factorial confirmatorio de la versión adaptada al español de la escala de apoyo social de Lubben-6.

- Todas los errores de medida y las cargas factoriales estandarizadas fueron significativas (tuvieron valores $t$ por encima de 1,96 como es recomendado) 22 .

La fiabilidad compuesta de ambos factores $(0,83$ y 0,91) y la varianza media extractada $(0.62$ y 0.75$)$ tuvieron valores satisfactorios $\underline{22}$.

Con respecto a la validez convergente, todas las cargas factoriales estandarizadas fueron mayores que 0.6 o 0.7 y estadísticamente significativas (valores de $T$ mayores a 1.96), coincidiendo con las recomendaciones de la bibliografía 22

La validez discriminante resultó óptima ya que la varianza media extractada de cada factor fue mayor que el cuadrado de la correlación entre los factores $(0,1521) \underline{23}$.

\section{Validación de criterio externo convergente}

Observamos una adecuada correlación Pearson $(0,67 ; p<0,001)$ entre el resultado del puntaje de Lubben y el puntaje asignado por el orientadora socio-sanitaria $\underline{24}$.

\section{Discusión}

Obtuvimos una versión en español de la escala Lubben-6 que fue validada en el ámbito ambulatorio y domiciliario en una muestra de 150 personas. Este instrumento mostró una adecuada validez de constructo y de criterio externo convergente, empleando como prueba de referencia para esta última a una evaluación profunda de 90 minutos de duración realizada en el domicilio del paciente por un equipo sociosanitario entrenado a tal fin.

Los indicadores de buen ajuste de la escala presentaron valores adecuados para el modelo de dos factores, excepto el RMSEA, que mostró un valor marginal (0.085). Esto podría estar explicado por que trabajamos con un tamaño muestral pequeño y constituye una limitación de nuestro trabajo. Sin embargo, algunos autores como Brown 19 restan importancia a este tipo de valor marginal del RMSEA cuando ocurre en el contexto en el que el resto de los valores se encuentran en el rango de lo recomendado.

Vale destacar que las validaciones del LSNS-6 realizadas en otros idiomas sólo habían evaluado la validez de constructo de la escala traducida y adaptada transculturalmente. Uno de los elementos más novedosos de nuestro trabajo es haber realizado también una validación a través de un criterio externo convergente, documentando buena correlación entre el puntaje de la escala LSNS-6 y una evaluación profunda realizada en el domicilio del paciente por la orientadora socio-sanitaria previamente entrenada a tal fin.

Respecto del idioma español, la única experiencia previa es la de Vilar-

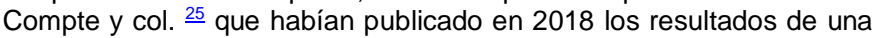
evaluación de la validez aparente en población mexicana de una versión del LSNS-6 traducida a dicho idioma, sin haber evaluado la validez de constructo ni la validez de criterio externo de dicha versión en español. En dicha publicación, estos autores comunicaron dificultades de comprensión en la población "blanco" respecto de los términos "parientes" y "amigos" (traducciones de relatives y friends del original en inglés). En el caso de pariente, las personas entrevistadas de la comunidad mexicana evocaban personas con un parentesco lejano, mientras que por familiar (otra posible traducción de relatives) entendían personas con parentesco muy cercano. En el caso de amigos también hubo problemas en lo referido a la cercanía (sólo se llamaba amigo a alguien muy cercano), pero merece la pena destacar también la dificultad que tuvieron con el género. En inglés, friends incluye tanto a amigos como amigas. En español, por convención, suele usarse al masculino como género que abarca ambos sexos. Sin embargo, algunas entrevistadas de la comunidad mexicana entendían literalmente el término y no en el sentido general, por lo que por amigos entendían exclusivamente personas de sexo masculino y por tanto, excluían de su evaluación a las amigas que formaban parte de su red social.

Nosotros no encontramos dificultades significativas respecto de la interpretación en Argentina del significado de las palabras amigo y familiar (en reemplazo de pariente), una vez que decidimos aclarar al inicio de la escala a qué nos referíamos con ambas palabras. Además, constatamos que en Argentina la interpretación que se le da a estos términos es más general y extensiva que en México, dado que por familiar se interpretada a toda persona que tuviera algún parentesco con el entrevistado, independientemente de que este fuera cercano o lejano. Respecto del término genérico "amigo", este evocó sin problemas a personas de cualquier género, quizás ayudado por la aclaración al inicio de la escala de que podíamos considerar amigo a toda persona con quien que no tuviera un parentesco pero que el entrevistado considerara cercana en términos de formar parte de su red social. Destacamos también que la correcta interpretación de las preguntas fue posteriormente reafirmada por los resultados obtenidos en el análisis de la validez de constructo.

Dado que el español es una de las lenguas más habladas en el mundo, consideramos valioso haber traducido, adaptado transculturalmente y validado el LSNS-6 al español. Sin embargo, entendemos que existen diferencias lingüísticas e interpretativas entre las diferentes comunidades hispanohablantes, por lo que consideramos que su aplicación en otros países distintos de Argentina debería ser sometida previamente, por lo menos, a una adaptación transcultural.

\section{Conclusión}

La versión traducida y adaptada transculturalmente al español de la escala LSNS-6 es válida y confiable, por lo que puede ser utilizada para la evaluación de la red de apoyo social en nuestra población anciana.

\section{Limitaciones de responsabilidad}

La responsabilidad del trabajo es exclusivamente de los autores.

\section{Conflictos de interés}

Ninguno

\section{Fuentes de apoyo}

No poseen fuentes de apoyo y de financiación.

\section{Originalidad del trabajo}

Este artículo es original y no ha sido enviado para su publicación a otro medio de difusión científica en forma completa ni parcialmente.

\section{Cesión de derechos}

Los participantes de este trabajo ceden el derecho de autor a la Universidad Nacional de Córdoba para publicar en la Revista de la Facultad de Ciencias Médicas y realizar las traducciones necesarias al idioma inglés.

\section{Participación de los autores}

Todos los autores han participado en la concepción del diseño, recolección de la información y elaboración del manuscrito, haciéndose públicamente responsables de su contenido y aprobando su versión final. 


\section{Bibliografía}

1. Holt-lunstad, J. \& Smith, T. Social Relationships and Mortality Risk: A Meta-analytic Review. SciVee (2010) doi:10.4016/19911.01.

2. House, J. S. Social isolation kills, but how and why? Psychosomatic medicine vol. 63 273-274 (2001).

3. Rizzuto, D., Orsini, N., Qiu, C., Wang, H.-X. \& Fratiglioni, L. Lifestyle, social factors, and survival after age 75: population based study. BMJ 345, e5568 (2012).

4. Dickens, A. P., Richards, S. H., Greaves, C. J. \& Campbell, J. L. Interventions targeting social isolation in older people: a systematic review. BMC Public Health 11, 647 (2011).

5. Cacioppo, J. T., Hughes, M. E., Waite, L. J., Hawkley, L. C. \& Thisted, R. A. Loneliness as a specific risk factor for depressive symptoms: cross-sectional and longitudinal analyses. Psychol. Aging 21, 140-151 (2006).

6. Cornwell, E. Y.\& Waite, L. J. Social Disconnectedness, Perceived Isolation, and Health among Older Adults. Journal of Health and Social Behavior vol. 50 31-48 (2009).

7. Hawkley, L. C., Thisted, R. A., Masi, C. M. \& Cacioppo, J. T. Loneliness predicts increased blood pressure: 5-year cross-lagged analyses in middle-aged and older adults. Psychol. Aging 25, 132-141 (2010).

8. Berkman, L. F., Glass, T., Brissette, I. \& Seeman, T. E. From social integration to health: Durkheim in the new millennium. Soc. Sci. Med. 51, 843-857 (2000).

9. Michael, Y. L., Berkman, L. F., Colditz, G. A. \& Kawachi, l. Living arrangements, social integration, and change in functional health status. Am. J. Epidemiol. 153, 123-131 (2001).

10. Cantero, M. C. T., López-Roig, S. \& López, M. C. N. Apoyo social e instrumentos de evaluación: revisión y clasificación. Anuario de psicología (2004).

11. Lubben, J. \& Gironda, M. Centrality of social ties to the health and well-being of older adults. Social work and health care in an aging society 319-350 (2003).

12. Lee, K. W. et al. The Validity and Reliability of Korean Version of Lubben Social Network Scale. Korean J. Fam. Med. 30, 352-358 (2009).
13. Ribeiro, O. et al. Versão portuguesa da escala breve de redes sociais de Lubben (LSNS-6). Revista Kairós: Gerontologia 15, 217-234 (2012).

14. Lubben, J. et al. Performance of an abbreviated version of the Lubben Social Network Scale among three European community-dwelling older adult populations. Gerontologist 46, 503-513 (2006).

15. Shioda, A., Tadaka, E. \& Okochi, A. Reliability and validity of the Japanese version of the Community Integration Measure for community-dwelling people with schizophrenia. Int. J. Ment. Health Syst. 11, 29 (2017).

16. Burnette, D. \& Myagmarjav, S. Translation and validation of the 18-item Lubben Social Network Scale with older adults in Mongolia. Int. Psychogeriatr. 25, 1493-1502 (2013).

17. Wild, D. et al. Principles of Good Practice for the Translation and Cultural Adaptation Process for PatientReported Outcomes (PRO) Measures: Report of the ISPOR Task Force for Translation and Cultural Adaptation. Value in Health vol. 8 94-104 (2005).

18. Streiner, D. L., Norman, G. R. \& Cairney, J. Health Measurement Scales: A Practical Guide to Their Development and Use. (Oxford University Press, 2015).

19. Brown, T. A. Confirmatory Factor Analysis for Applied Research, Second Edition. (Guilford Publications, 2015).

20. Finney, S. J. \& DiStefano, C. Non-normal and categorical data in structural equation modeling. Structural equation modeling: A second course 10, 269-314 (2006).

21. Hair, J. F. Multivariate Data Analysis: An Overview. International Encyclopedia of Statistical Science 904-907 (2011) doi:10.1007/978-3-642-04898-2_395.

22. Hair, J. F., Black, W. C., Babin, B. \& Anderson, R. E. Multivariate data analysis . Cengage. (2018).

23. Fornell, C. \& Larcker, D. F. Evaluating Structural Equation Models with Unobservable Variables and Measurement Error. Journal of Marketing Research vol. 18 39-50 (1981).

24. Streinmer, D. L. A Checklist for Evaluating the Usefulness of Rating Scales. Can. J. Psychiatry 38, 140-148 (1993).

25. Vilar-Compte, M., Vargas-Bustamante, A. \& Lubben, J. Validation Study of the Abbreviated Version of the Lubben Social Network Scale Spanish Translation among Mexican and Mexican-American Older Adults. J. Cross. Cult. Gerontol. 33, 83-99 (2018). 
Escala LUBBEN-6 para red de apoyo social

\section{FAMILIA}

(Considerando como tal a las personas con las que usted tiene algún parentesco)

1. ¿Con cuántos familiares tiene usted contacto personal o telefónico al menos una vez por mes?

$0=0$ puntos

$1=1$ punto

2 = 2 puntos

$3 \circ 4=3$ puntos

de 5 a $8=4$ puntos

9 o más $=5$ puntos

2. ¿Con cuántos familiares se siente usted cómodo para conversar con facilidad sobre los asuntos privados que a usted le preocupan?

$0=0$ puntos

$1=1$ punto

$2=2$ puntos

$3 \circ 4=3$ puntos

de 5 a $8=4$ puntos

9 o más $=5$ puntos

3. ¿A cuántos familiares los siente usted lo suficientemente cercanos como para llamarlos en caso de necesitar ayuda?

$0=0$ puntos

$1=1$ punto

$2=2$ puntos

$3 \circ 4=3$ puntos

de 5 a $8=4$ puntos

9 o más $=5$ punto

\section{AMIGOS}

(Considerando como tales a aquellos con quienes tiene algún vínculo pero no son familiares)

4. ¿Con cuántos amigos tiene usted contacto personal o telefónico al menos una vez por mes?

$0=0$ puntos

$1=1$ punto

$2=2$ puntos

$3 \circ 4=3$ puntos

de 5 a $8=4$ puntos

9 o más $=5$ puntos

5. ¿Con cuántos amigos se siente usted cómodo para conversar con facilidad sobre los asuntos privados que a usted le preocupan?

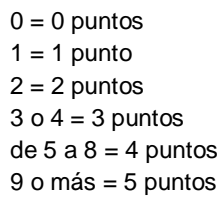

6. ¿A cuántos amigos los siente usted lo suficientemente cercanos como para llamarlos en caso de necesitar ayuda?

$0=0$ puntos

$1=1$ punto

$2=2$ puntos

$3 \circ 4=3$ puntos

de 5 a $8=4$ puntos

9 o más $=5$ puntos

\begin{tabular}{|l|l|l|l|l|l|l|}
\hline Preg. 1 & Preg. 2 & Preg. 3 & Preg. 4 & Preg. 5 & Preg. 6 & TOTAL \\
\hline & & & & & \\
\hline
\end{tabular}

